\title{
EXPERIENCING STUDENTS' DIFFICULTIES IN LEARNING ABSTRACT ALGEBRA
}

\author{
Abatar Subedi \\ Lecturer, Department of Mathematics Education, Central Department of Education, \\ Kirtipur, $T U$. \\ Correspondingauthor: abatar.subedi@tucded.edu.np
}

\begin{abstract}
This paper aims to reveal the difficulties as experienced by graduate students while learning abstract algebra at master's degree in mathematics education in the first month of their enrollment. For, I adopted a case study of five students, interviewed them with the help of interview guidelines and observed their behaviors in the classroom, and triangulated this information with researcher's experiences to explore the difficulties in learning abstract algebra. I used inductive method to analyze the information and concluded that graduate students have experienced several difficulties in learning abstract algebra including the difficulties in conceptualizing algebraic facts, constructing examples and non-examples; and proving theorems. Finally, the study suggests that graduate teaching need to focus on conceptual and procedural understanding of students, and emphasizing to construct examples and non-example as much as possible to improve students learning.
\end{abstract}

Keywords: Abstract algebra - conceptualizing facts - constructing examples proving theorems

\section{INTRODUCTION}

Algebra is one of the fundamental courses of mathematics included in the course of school level to higher education. School algebra is a generalization of arithmetic where the variables are the numbers, but abstract algebra is the generalization of school algebra (Findell, 2001). Similarly, Stacey and Chick (2004) defined algebra as: "a study of symbol manipulation, equation solving and functions; a way of expressing generality, to solve certain classes of problems; to model the real situations; and a formal system involving set theory, logic and operations of entities, 
and real numbers. Likewise, Booth (1986) argued that the main purpose of algebra is to learn how to represent general relationship and procedures, through these representations, a wide range of problems can be solved and new relationship can be developed from those known.

But the graduate abstract algebra includes algebraic structures which is the set together with the binary operation defined on it and several properties of these algebraic structures: groups, rings, fields, vector spaces and modules. It also includes the concepts of homomorphism, isomorphism and other linear transformations. Learning abstract algebra at there is to understand abstract algebraic structure, its notations, symbols and their relationships logically. In particular, students need to memorize facts, notations, symbols, axioms, definitions; construct examples and nonexamples; prove statements theoretically as well as numerically by applying knowing facts together with connecting established relations. As explained by Fry et al. (2009), learning

is not a single thing; it may involve mastering abstract principles, understanding proofs, remembering factual information, acquiring methods, techniques and approaches, recognition, reasoning, debating ideas, or developing behavior appropriate to specific situation; it is about change (p.8).

Similarly, Lalonde (2013) stated that graduate students have to "think of examples that satisfy the definition and the next to think about why the definition is useful, why we are bothering to make this definition, and why it does say what it is, for meaningful understanding to abstract concept (p.23). Understanding proving skills of algebraic statements is an important skills required for graduate students (Judson \& Austin, 2015, p.2) which requires strong conceptual and procedural skills of contents domain. Thus, to understand it, students need to have capability of selecting appropriate method of proofs where proofs in algebra is understood as "a convincing and logical argument, derived from previously known facts, about the accuracy of the mathematical statement" (Judson \& Austin, 2015, p.1). Thus, one who cannot apply known facts to prove the new ones and do not have capability of handling axioms, definitions, and arguments suffer from difficulty to learn abstract algebra.

Understanding notations, symbols, and language of algebra; constructing examples, non-examples and counter examples are the necessary skills for graduate students. To support this argument Judson and Austin, 
(2015) stated that students' have some common difficulties in learning new concepts due to poor knowledge in mathematical quantifiers, cannot choose appropriate methods for proving theorems and cannot understand the given hypothesis (pp.2-3). So, how graduate students experienced, encountered, suffered and tackled from difficulties while learning abstract algebra during the first month of higher education at master level was therefore considered a research theme.

\section{OBJECTIVE}

The objective of this study was to explore the difficulties of graduate students in learning abstract algebra at master's level of mathematics education.

\section{LITERATURE REVIEW}

The nature of concepts can play an important role in learning and understanding. For, Lalonde (2013) pointed that the concepts in calculus, geometry and linear algebra can be viewed with lots of concrete examples and numerical computations, but the concepts in graduate abstract algebra are more abstract in nature which will take a much more general approach to conceptualize (p.4). The abstraction of concepts influences in proving theorem which is "one of the major tasks for graduate students in algebra" (Judson \& Austin, 2015). Students need to select appropriate techniques of proofs: "direct proofs, proof by contradiction, induction, and proof by contrapositive" (Lalonde, 2013), otherwise they face difficulties. Proofs are "convincing arguments about the accuracy of the mathematical statement" (Judson \&Austin, 2015), derived from previously known definitions, facts and statements. The algebraic arguments are highly valued by students but difficult to produce or understand (Nardi \& Iannone, 2006).

Some common difficulties to learn new concepts are due to poor knowledge in mathematical quantifiers, unable to select appropriate methods and not understanding given hypothesis(Judson, \& Austin, 2015). Because of poor knowledge/skills in symbolic language, students have faced difficulties in learning algebra I (Nathan \& Koedinger,1999 -2003 ), and the origin of errors are due to intuitive assumptions, failure to understand the syntax of algebra, analogies with other familiar symbol system (Egodawatte, 2009) at school. Concrete models like examples and other materials (Witzel, 2005), and strong in pre-requisites knowledge are keys to improve conceptual knowledge in algebra which helps students to increase the procedural knowledge (Booth \& Koedinger, 2008). 
Likewise, focus on building capacity of conceptual understanding is important than focus on only memorizing the solving procedure in abstract algebra (Zuya, 2017).Explaining examples, non-examples either alone or in combination of them is beneficial to improve conceptual understanding (Booth et al., 2013). The abstraction levels are understood as: quality of the relationships between the object of thought and the thinking person, reflection of the process-object duality, and the degree of complexity of the concepts of thought (Hazzan, 1999). That means if students cannot understand these abstraction level which will produce difficulties in learning.

But recently, the mathematics learning at higher level is understood in terms of APOS (action, process, object and schema)framework which was developed by Dubinsky and McDonald (2001). The action is understood as a transformation of objects perceived by student with the help of external stimuli or step-by-step instructions on how to perform the operation.

By the repetition of actions and reflection on it, student can make an internal mental construction called process by which student can think of as performing the same kind of action with little support of external stimuli. If the student become totally aware from the process and realize that transformations can act on it then object conception is constructed. Finally, the schema is the combination of actions, process and objects for certain mathematical concepts in the mind, and understood the concepts totally as an object. Thus, there may be difficulties of graduate student to develop the conceptions of each level accordingly in learning abstract algebra.

\section{METHODOLOGY}

I used constructivist research paradigm which believes on relativist ontology, subjective epistemology and qualitative methodology(Guba \& Lincoln, 2005)in which the researcher "aim to get a deeper understanding of the phenomenon and its complexity in its unique contexts, not to generalize to a whole population" (Creswell, 2009). This means reality is socially constructed, local and specific; and researcher and respondents both involve in knowing process (Fard, 2012). I selected only five students purposively who showed poor performance in understanding abstract algebra at master level. I used semi-structured interview guidelines and observation notes to gather information. The difficulties as experienced by selected students in learning abstract algebra are the information of this study.I analyzed the 
information by using grounded theory (Strauss \& Corbin, 1998) and general inductive approachas explained by Thomas (2006).

\section{RESULT AND DISCUSSIONS}

The results were presented and discussed under theseparate headings as below.

\section{Difficulties in conceptualizing facts}

Conceptualizing facts in algebra means to understand symbols, terminologies, definitions, facts, mathematical language, and algebraic structures and reasoning. Understanding of mathematical reasoning requires familiarity with the precise meaning of words used in mathematics (every, for some, not, and, or) and relevant aspects of language (word order, quantifiers, logical statements and logical symbols (D'Angelo et al., 2000). For example, binary operation is one of the fundamental concepts in abstract algebra. It is defined as: an operation *: $G \times G \rightarrow G$ is said to be a binary operation in a non-empty set $G$ if $a * b \in G$ for every $a, b$ in $G$. It was experienced that majority of students defined this concept in the class of master degree in mathematics education.

However, they were not able to explain the detail meaning of the symbol * . Frankly speaking, three selected students understood this symbol represent just either addition(+) or multiplication (.), and two students understood it as just multiplication in the non-empty set $G$. They were not informed this symbol represent variable like: in the set of function it will represent 'composition', in the set of sets it will represents 'union', in the set of matrix it will represent matrix addition, matrix multiplication and so forth. Due to poor understanding of mathematical meaning of the symbol*, it was difficult to defined that 'binary operation' is just a function $f: G \times G$ $\rightarrow$ Ggiven by $(a, b) \mapsto a f b$ for all $a, b$ in $G$ where the function $f$ is the binary operation.Particularly, addition and multiplication are binary operations in the sets $\mathbf{N}$ of natural numbers, $\mathbf{Z}$ of integers, $\mathbf{Q}$ of rational numbers, $\mathbf{R}$ of real numbers and in the set $\mathbf{C}$ of complex numbers. However, subtraction is not binary operation in the set $\mathbf{N}$.

Without knowing binary operations, it was experienced that students had difficulties in understanding algebraic concepts: algebraic structure, semi-group, monoid, group, abelian group, permutation group and cyclic 
groups in abstract algebra. One of the examples was as follows. When I asked students about the concept of cyclic group then they defined it as:

"A group $G$ is said to be cyclic if every element of $G$ can be expressed as the various power of the single element of $G$ and other defined as: the group generated by single element is called cyclic group. This single element is called the generator". After that I asked one example of cyclic group, then most of them replied "the multiplicative group $G=\left\{1, w, w^{2}\right\}$ where $w$ is one of imaginary root of unity is the cyclic group because every element of $G$ can be expressed as the different power $w, w^{2}, w^{3}=1$ " of $w$. Another examples of cyclic groups they gave me were $G=\{1,-1, i,-i\}$ where $i^{2}=-1$ and $H=\{1,-1\}$ under multiplication as binary operation. These responses show that definition and example of the cyclic group were correct. But I was not conformed that whether they have good concepts of cyclic group or not? Again I asked them that what about the group of integer $\mathbf{Z}$ ? One of the student replied me "it is also a cyclic group generated by 1 " but did not able to express every element as the power of 1 . Most of them expressed $1^{0}=1,1^{1}=1,1^{2}=1 \ldots$ in the group of integers.

From this response I experienced that graduate students did not have good understanding of cyclic group due to poor concepts of binary operations. Since $\mathbf{Z}$ is the group under addition then $1^{2}=1+1$ (adding two times 1$)=2,1^{3}=1+1+1=3($ adding three times 1$), \ldots$ implies $1^{0}=0$ (adding zero times 1 ). Similarly, $1^{-1}=-1$ implies $1^{-2}=\left(1^{-1}\right)^{2}=-2$ and so on. In general, $a^{n}$ means $a+a+\ldots+a=n a$ for every integer $n$ in $\mathbf{Z}$. Thus, graduate students have difficulties in conceptualizing facts to learn abstract algebra, even who can define the concepts correctly.

\section{Difficulties in constructing examples}

Examples and non-examples are the keys to conceptualize in abstract algebra. For meaningful understanding the concepts in abstract algebra "we immediately try to think of examples that satisfy the definition and the next to think about why the definition is useful, why are we bothering to make this definition, and why does it say what it is"(Lalonde, 2013, p.13). Explaining worked examples, and incorrect examples (non-examples) either alone or in combination with correct examples are beneficial for conceptual understanding in algebra (Booth et al., 2013). 
By nature of concepts in algebra, it is sometime more difficult to construct the related examples even for experts. For example, the definition of group in abstract algebra is as: a non-empty set $G$ together with the operation * is said to be a group if the set $G$ satisfying the closure axiom, associative axiom, existence of identity and existence of inverse of each element of $G$ in $G$ under the operation *. This definition of group is abstract in nature. Almost all selected students defined this concept of group. But when I asked an example of it, only two students out of selected gave an examples themselves. One of which was the set of integer $\mathbf{Z}$ under addition is a group. It is group because the sum of any two integers is an integer, the zero integer act an identity, the inverse of $a$ in $\mathbf{Z}$ is $-a$ in $\mathbf{Z}$ and $\mathbf{Z}$ holds associative property of addition.

When I asked to test group properties in $\mathbf{Z}, \mathbf{Q}, \mathbf{R}$, and $\mathbf{C}$ under multiplication, then students only replied that $\mathbf{Z}$ is not a group because 2 has not multiplicative inverse in $\mathbf{Z}$ and rest are groups. The rest are not also groups because the zero element in them has no multiplicative inverse. If we remove zero from the sets of rationales, reals and complex number then they satisfy the group properties under multiplication with identity element 1. Similarly, I asked students to find a group in which multiplicative identity is other than 1 . The students did not able to construct such examples. They felt that "it was more difficult to construct". I constructed one example of it as: let $G$ be a set of all non-zero rational numbers where the binary operation is defined: $a^{*} b=a b / 3$ then $G$ is a group with identity element 3 . Here 3 act as a role of 1 in multiplication. That is why the nature of concepts including in abstract algebra itself create difficulty in constructing examples.

Moreover, I asked students about the concept of 'abelian' and 'non-abelian' groups. Then they defined as: a group $G$ is called an abelian if $a * b=b^{*}$ a for every $a, b$ in $G$, otherwise it is non-abelian. Only one students gave the example of non- abelian group which was the set $G=$ set of all invertible $n \times n$ matrices having real entries under multiplication of matrices. It is non-abelian because matrix multiplication is not commutative in general. But rest of the students only did give example of abelian group, the set of integer together with addition.

Similarly, selected students defined the concepts of permutation groups, simple groups, group homomorphism, group isomorphism, 
subgroups and normal subgroups. However, it was experienced in the classroom teaching that they were unable to construct suitable examples of them. Thus, it is concluded that the graduate students are still facing difficulties in constructing suitable examples and non-examples even for the fundamental concepts in abstract algebra.

\section{Difficulties in proving theorems}

Proving theorems (Judson \& Austin, 2015) and selecting appropriate techniques (direct proof, induction, proof by contradiction, proof by contrapositive) of proofs (Lalonde, 2013) in abstract algebra are required skills for graduate students. The mathematical proofs are the convincing arguments mainly derived from previously known definitions, facts and proved statements about the accuracy of the mathematical statement (Judson \& Austin, 2015).

One of my participant expressed as "I have faced difficulty on how to start proof and where to start it, sometime it starts with definition and some time it is not direct. For example, to show the existence of unique element in a group, we assume two identities $e, e$ ' in the group $G$ and then prove $e=e$ '. But to prove the theorem like Lagrange's theorem (If $G$ is a finite group of order $n$ and $H$ be its subgroup with order $m$ then $m$ divides $n$ ), we need different techniques".

For assume $a_{1} H, a_{2} H, \ldots, a_{r} H$ be distinct cosets of $H$ in $G$ then $G$ $=a_{1} H \cup a_{2} H \cup \ldots \cup a_{r} H$ then $\mathrm{o}(G)=\mathrm{o}\left(a_{1} H\right)+\mathrm{o}\left(a_{2} H\right)+\ldots+\mathrm{o}\left(a_{r} H\right)$. This implies that $n=\mathrm{o}(H)+\mathrm{o}(H)+\ldots+\mathrm{o}(H)(r$ times $)$, and so $n=m r$ implies $m$ divide $n$. In the first type of proof students need to assume two possibilities and use group axioms to show the uniqueness of identities. But in the proof of Lagrange's theorem, there are several logical arguments which are based on previously known concepts such as order of finite groups, cosets, distinct cosets, group partitions and other general rules of mathematics. According to APOS theory (Dubinsky\& McDonald, 2001), students need to have object conception of Lagrange's theorem where the individual become aware of the process as a totally and relies the transformation on it.

The other participant expressed his difficulty as "I have difficulties to connect the known facts in logical arguments of the proof even I know what is given and what need to be prove as in Lagrange's theorem. Here, I know to prove required to show the order of subgroup should divide order of finite group, but it is very difficult me, where and how to start to prove it". These responses indicate that there is difficult to select correct method and 
to connect known facts in logical sequence for proving algebraic statement in the graduate level. That is graduate students have both procedural and conceptual difficulties in proving theorems.

Understanding the language and meaning of terminologies in the proof of algebraic statements is another aspects of difficulty. One of my participant reported that "I am very confusing whether it need to show well defined condition or not in proving isomorphism theorems of the groups. However, I know the concepts homomorphism, isomorphism and bijective function. Also I feel difficulty to show onto in proving isomorphism theorems". This response implies that the graduate students have difficulties according to nature of concepts involved in the proof. Dubinsky and McDonald (2001) theory's also supports that there are four level of conceptions: action, process, object and schema of abstract mathematics and so students' difficulties are varied according to their understanding level of algebraic concepts.

Furthermore, the participants replied that "we sometime have faced difficulties in understanding the meaning of algebraic symbol and operations". Their experience explain that they are confusing the "meaning of double vertical lines $(||)$, tilde $(\sim)$, equivalence $(\cong)$ and other mathematical quantifier such as for all, for some, not for all, consequently and so forth". These explanations show that students have faced difficulties to understand the conditional meaning of symbols (mathematical meaning) and quantifiers in abstract algebra. For example, the double vertical line indicates the meaning of order in group, absolute value for real numbers and determinant for square matrix.

\section{CONCLUSION}

Learning abstract algebra is to memorize facts, definitions, symbols; understand algebraic language, structures, operations; construct examples, non-examples; solve problems; and prove theorems. The graduate students have faced difficulties in conceptualizing such concepts; to construct related examples and non-examples; to select correct approaches of proving, and to understand overall logical relationships, structures and arguments in learning abstract algebra. That means they have both procedural and conceptual difficulties as explained by Zuya, (2017) and which are due to poor capability of reducing the abstraction level as pointed in Hazzan (1999) in abstract algebra. Also, these difficulties are similar to difficulties in 
learning action, process, object and schema conceptions of abstract algebra as explained in APOS learning framework of (Dubinsky \& McDonald, 2001). Finally, the study suggests that graduate teaching have to focus on constructing examples and non-examples for conceptual and procedural clarity of abstract algebraic concepts.

\section{REFERENCES}

Booth, L.R. (1986). Difficulties in algebra. Australian Mathematics Teacher, 42(3): 2-4.

Booth, J.L., \& Koedinger, K.R.(2008). Key misconception in algebraic problem solving. http://pact.cs.cmu.edu/pubs/ BoothKoedingerCogSci2008.pdf

Booth, J.L., Lange, K.E., Koedinger, K.R., \& Newton, K.J. (2013). Example problems that improve student learning in algebra: Differentiating between correct and incorrect examples. Learning and Instruction, 25:24-34.

Creswell, J. W. (2009). Research design: Qualitative, quantitative, and mixed methods approaches (3rd ed.). Sage Publication, Inc.

D' Angelo, J.P.,\& West, D. B. (2000). Mathematics thinking: Problemsolving and proof (2nd ed.). Prentice Hall Inc.

Dubinsky, E., \& McDonald, M.A. (2001). APOS: A constructivist theory of learning in undergraduate mathematics education research. https://pdfs.semanticscholar.org/6850/ b01648bf43e15cbdbcf871b215c3cf44825d.pdf

Egodawatte, G. (2009). Is algebra really difficult for all students? Acta Didactica Napocensia, 2(4).

Fard, H.D. (2012). Research paradigm in public administration. International Journal of Humanities, 19(4): 55-108.

Findell, B. R. (2001). Learning and understanding abstract algebra $(\mathrm{PhD}$ Dissertation), University of New Hampshire. University of New Hampshire Scholar's Repository. https://scholars.unh.edu/cgi/ viewcontent.cgi? article $=1050 \&$ context $=$ dissertation

Fry, H., Ketteridge, S., \& Marshall, S. (2009). Understanding student learning. In: H. Fry, S. Ketteridge \& S. Marshall (eds.), A handbook for teaching and learning in higher education enhancing academic practice (3rd ed., pp. 8-26). Routledge. 
Guba, E.G. \& Lincoln, Y.S. (2005). Paradigmatic controversies, contradiction, and emerging confluences. In: N.K. Denzin \& Y. S. Lincoln (eds.), The SAGE handbook of qualitative research (3rd ed., pp.191-215). Sage.

Hazzan, O. (1999). Reducing abstraction level when learning abstract algebra concepts. Journal of Educational Studies in Mathematics, 40 (1): 71-90.

Judson, T.W.,\&Austin, S.F.(2015). Abstract algebra: Theory and application. http://mathbook.pugetsound.edu

Lalonde, S. M. (2013). Notes in abstract algebra. https://math.dartmouth. edu/archive/m31x13/public_html/Notes $\% 20$ on $\% 20$ Abstract $\% 20$ Algebra\%202013.pdf

Nardi, E.,\& Iannone, P. (2006). How to prove it: Abrief guide for teaching proof to year 1 mathematics undergraduates. UEA Print Service.

Nathan, M.J., \& Koedinger, K. (1999-2003). Teachers' judgments about algebra problem difficulty. Technical Report, Institute of Cognitive Science, University of Colorado, Boulder.

Stacey, K., \& Chick, H. (2004). Solving the problem with algebra. In: K. Stacey, H. Chick \& M. Kendal (eds.), The future of the teaching and learning algebra, the 12th ICMI study (pp. 1-20). Kluwer Academic Publishers.

Strauss, A., \& Corbin, J. (1998). Basics of qualitative research: Techniques and procedures for developing grounded theory. Sage Publications, Inc.

Thomas, D.R. (2006).A general inductive approach for analyzing qualitative evaluation data. American Journal of Evaluation, 27(2): 237-246.

Witzel, S.B. (2005). Using CRA to teach algebra to students with math difficulties in inclusive setting. Learning Disabilities: A Contemporary Journal, 3(2): 49-60.

Zuya, H.E. (2017). Prospective teacher' conceptual and procedural knowledge in mathematics : The case of algebra. American Journal of Educational Research, 5(3): 310-315. 